\title{
RECURSOS HUMANOS EN SALUD: UNA MIRADA AL INTERIOR DEL PERU
}

\section{HUMAN RESOURCES FOR HEALTH: A VIEW INSIDE PERU}

\author{
Oswaldo Salaverry ${ }^{1,2, a}$
}

En un país con la enorme diversidad geográfica y cultural del Perú, el personal del sector salud actúa necesariamente en muy diversos escenarios y en muy distintas circunstancias, las que abarcan desde los centros hospitalarios de alta complejidad hasta el trabajo de campo en el altiplano o en los ríos amazónicos. Las condiciones de trabajo, marcadas por estos condicionantes, exigen frecuentemente grandes esfuerzos para poder brindar servicios a la población. En la galería fotográfica que presentamos intentamos reflejar algo de la diversidad de ámbitos de actuación del personal de salud en Perú.

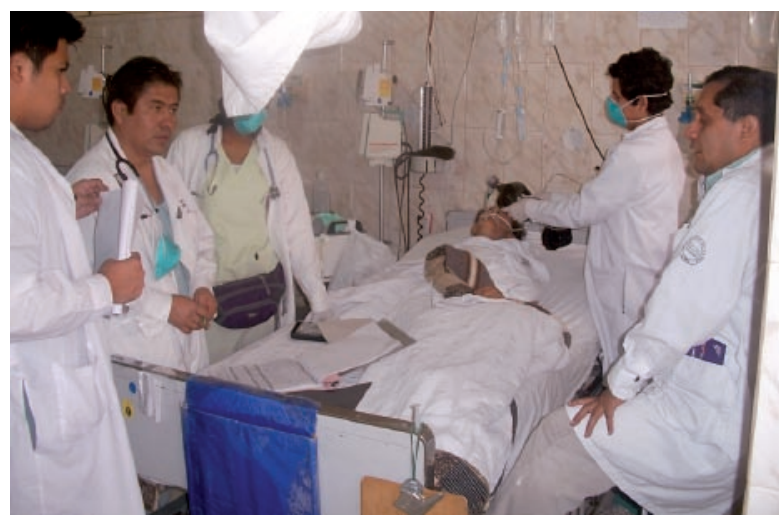

Foto 1. Discusión clínica a la vera del paciente en Hospital General de Lima (cortesía Dr. Alonso Soto).

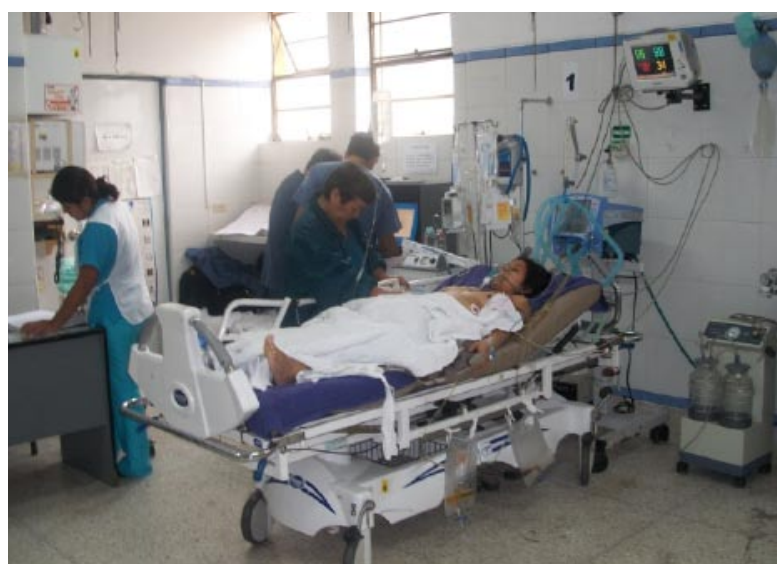

Foto 2. Médica y enfermeros en sala de trauma shock en Hospital General de Lima (cortesía Dr. Alonso Soto).

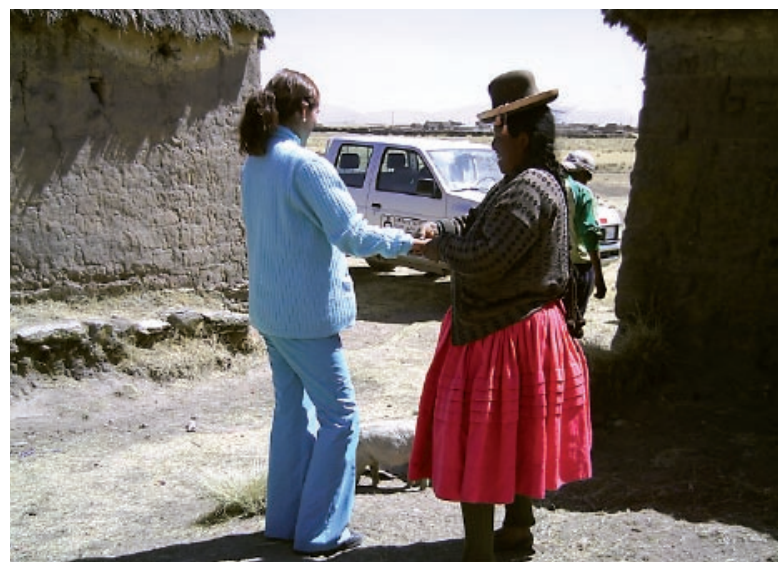

Foto 3. Enfermera de Puesto de Salud Checca en llave (Puno) coordinando con partera Aymara para atención de gestantes de la comunidad (cortesía R. Quispe. CENSI).

\footnotetext{
Centro Nacional de Salud Inercultural (CENSI), Instituto Nacional de Salud. Lima, Perú.

2 Departamento Académico. de Medicina Preventica y salud Pública, Facultad de Medicina, Universidad Nacional Mayor de San Marcos. Lima, Perú. a Médico; ${ }^{b}$ Doctor en Medicina.
} 


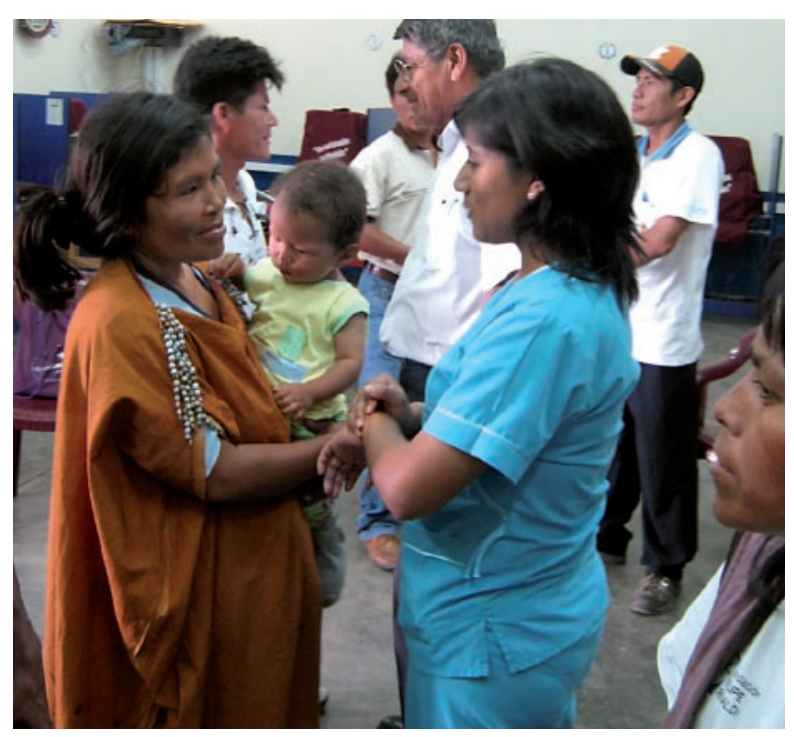

Foto 4. Diálogo con gestante ashaninka. El personal de salud debe desarrollar competencias lingüísticas y culturales para acercarse a comunidades de otras culturas. Centro de Salud Mazamari. Satipo Junín. (cortesía R. Quispe. CENSI).

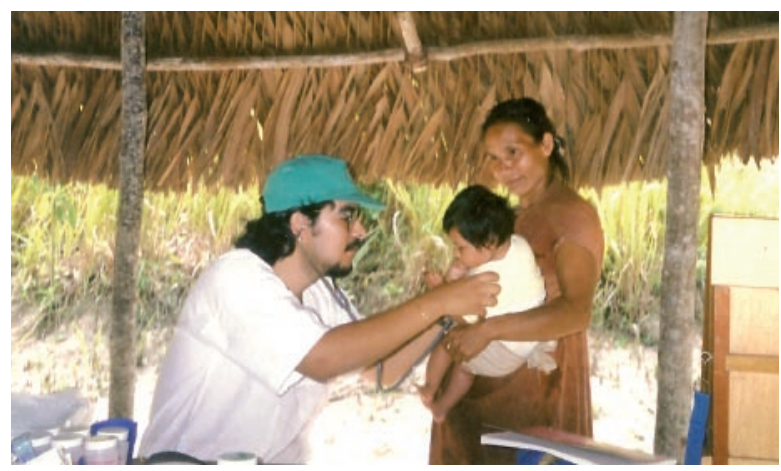

Foto 5. Médico del servicio rural atendiendo niño indígena. El primer contacto de la mayoría de profesionales de la salud con la realidad sanitaria y la diversidad cultural del país se realiza en el desarrollo de su servicio rural (cortesía Dr. Alonso Soto).

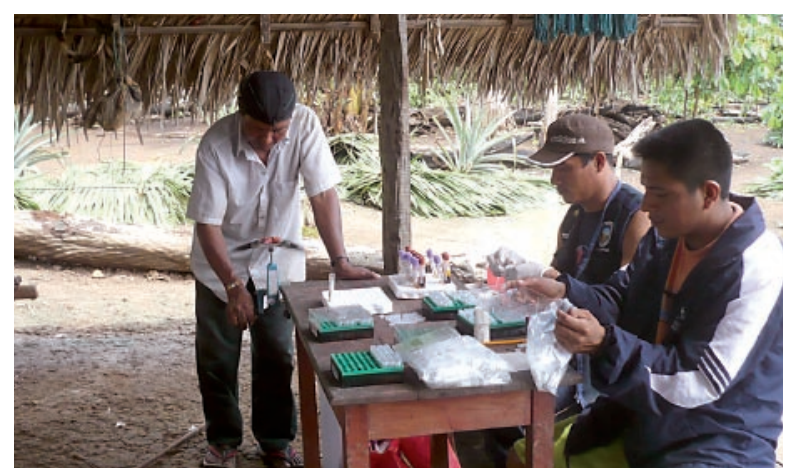

Foto 6. Personal de salud realizando pruebas de laboratorio durante trabajo de campo en vivienda de una comunidad amazónica (cortesía CENSI, INS).

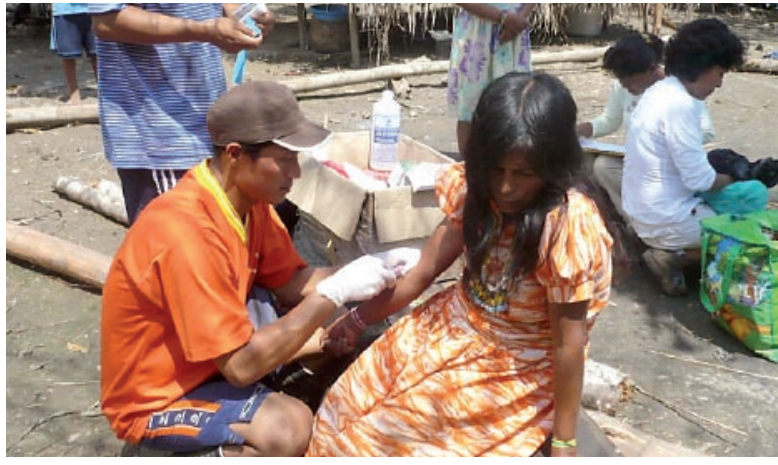

Foto 7. Toma de muestras sanguíneas en campaña de salud en comunidad amazónica. El personal de salud debe adaptarse a las condiciones ambientales y geográficas sacrificando muchas veces el uso del uniforme entre otras limitaciones (cortesía CENSI, INS).

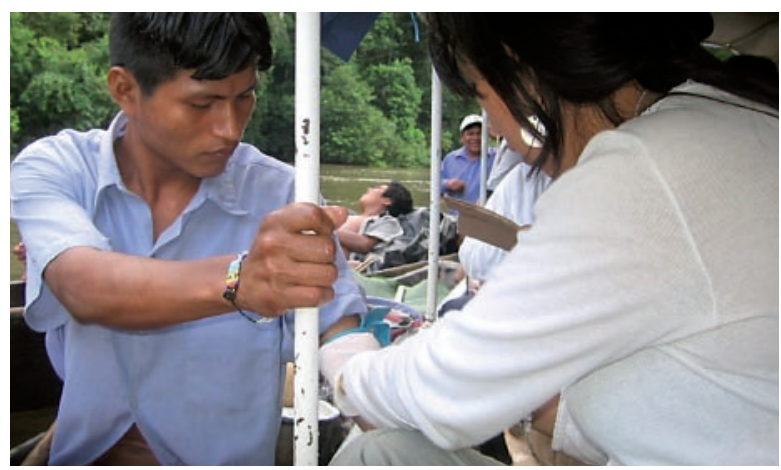

Foto 8. Personal de salud atendiendo desde pequeña embarcación, nótese el bote acoderado a babor con otro paciente esperando atención. La dispersión y aislamiento de las comunidades, especialmente en la amazonía aguza el ingenio de los servidores de salud para brindar atención (cortesía CENSI, INS).

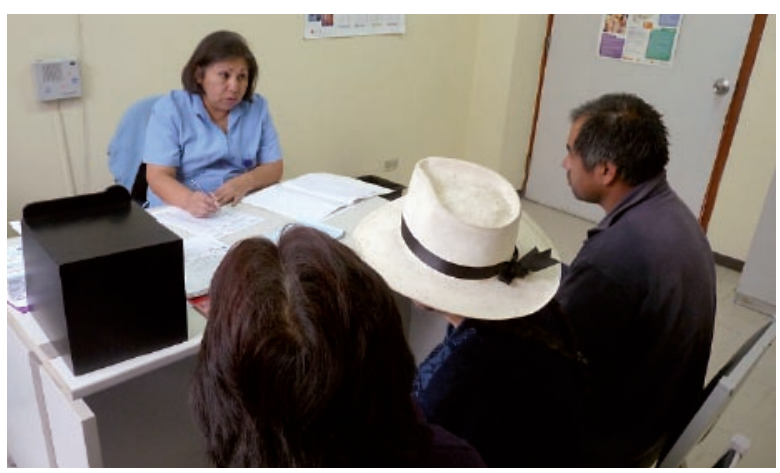

Foto 9. Consulta en servicio de psiquiatría de Hospital especializado a paciente andino de lengua materna quechua. La diversidad cultural impone retos y exige nuevas competencias al personal de salud en todos los escenarios sean urbanos o rurales, de alta complejidad o de atención primaria.

Correspondencia: Oswaldo Salaverry García

Dirección: Capac Yupanqui 1400, Lima, Perú.

Teléfono: (511) 617-6200 Anexo: 1609

Correo electrónico: osalaverry@ins.gob.pe 